\title{
4D Sonography in Prenatal Diagnosis of Fetal Anomalies in First and Second Trimester of Pregnancy
}

\author{
Ahmed F. Yousef, Osama T. Galal, Taghreed H. Atta
}

Department of radiology, Benha faculty of medicine, Benha University, Egypt

Correspondence to: Taghreed H. Atta, Department of radiology, Benha faculty of medicine, Benha University, Egypt

Email:

taghreedhossam2020@gmail.com

Received: 21 July 2020

Accepted: 28 July 2020

\begin{abstract}
:
Background: One of the most consistently used justifications for the use of obstetric ultrasound is that, accurate diagnosis of fetal malformations before delivery can provide health care providers and parents a number of management options. Aim: evaluate the role of 4D ultrasound in assessment and detection of fetal congenital anomalies, in 1 st \& 2nd trimester of pregnancy. Subjects \& Methods: Fifty women were examined by 2D followed by 4D US, and 52 detected anomalies. Results: Our results demonstrate an advantage of 4D US over 2DUS in $13 \%$ of cases, equal findings in $30 \%$, and less information in $17 \%$ of anomalies detected. Four dimensional US is advantageous in some anomalies of the face and extremities only. Conclusion: 2D US remains the gold standard in assessment of fetal anomalies, and 4D US, therefore, is not a screening technique but an adjunct to 2D US for those fetuses in whom malformations are already determined or suspected on the basis of standard sonography.
\end{abstract}

Keywords: 4D sonography; prenatal; anomalies

\section{Introduction}

Routine ultrasound examination is an established part of antenatal care if resources are available and access possible. It is commonly performed in the second trimester, although routine scanning is offered increasingly during the first trimester, particularly in high-resource settings. Ongoing technological advancements, including high-frequency transvaginal scanning, have allowed the resolution of ultrasound imaging in the first trimester to evolve to a level at which early 
fetal development can be assessed and monitored in detail (1).

In general, the main goal of a fetal ultrasound anomaly scan using 4D ultrasonography is, to provide accurate information which will facilitate the delivery of optimized antenatal care with the best possible outcomes for mother and fetus. In early pregnancy, it is important to confirm viability, establish gestational age accurately, determine the number of fetuses and, in the presence of a multiple pregnancy, assess chorionicity and amnionicity. Towards the end of the first trimester, the scan also offers an opportunity to detect gross fetal abnormalities and, in health systems that offer first-trimester aneuploidy screening, measure the nuchal translucency thickness (NT). It is acknowledged, however, that many gross malformations may develop later in pregnancy or may not be detected even with appropriate equipment and in the most experienced of hands (2).

The second-trimester '18-22-week' scan remains the standard of care for fetal anatomical evaluation in both low-risk and high-risk pregnancies. First-trimester evaluation of fetal anatomy and detection of anomalies was introduced in the late 1980s and early 1990s with the advent of effective transvaginal probes. The introduction of NT aneuploidy screening in the 11 to $13+6$-week window has rekindled an interest in early anatomy scanning. Reported advantages include early detection and exclusion of many major anomalies, early reassurance to at-risk mothers, earlier genetic diagnosis and easier pregnancy termination if appropriate. Limitations include need for trained and experienced personnel, uncertain cost/benefit ratio and late development of some anatomical structures and pathologies (e.g. corpus callosum, hypoplastic left heart), which make early detection impossible and can lead to difficulties in counseling due to the uncertain clinical significance of some findings. (2)

As sonography has become a routine component of prenatal care, many of these disorders are diagnosed prenatally confronting family and physician with the question of elective termination of pregnancy. Ideally, this question is discussed on the basis of a specific diagnosis. However, such a diagnosis is difficult to achieve by fetalsonography. Even under optimal conditions, it is missed in at least $35 \%$ of cases meaning that many abortions are performed on the basis of diagnostic suspicion. Postnatally, the prenatal diagnosis has to be verified (3).

To do that, fetal radiography becomes important. It is an effective, simple and economic way to establish a diagnosis or to narrow the number of diagnostic possibilities sufficiently to direct pathological, 
biochemical or molecular studies in their quest for a specific diagnosis. It provides the clinical basis for research. More importantly, it is required for proper parental counseling (4).

This study aimed to evaluate the role of $4 D$ ultrasound in assessment and detection of fetal congenital anomalies, in 1 st \& 2 nd trimester of pregnancy.

\section{Subjects and methods}

The study has been done on 50 high risk pregnant women (1st and 2nd trimester), during last year August 2018 till August 2019. This cross-sectional observational study, was approved by the ethical committee of Benha faculty of medicine

Some of patients were examined at their $1^{\text {st }}$ trimester, other were diagnosed in their $2^{\text {nd }}$ trimester as part of fetal anomaly scan. part of routine maternal care, suspected cases will advised for undergoing 4D ultrasonography and data were collected from Fetal Medicine Unit at Cairo University Hospital many private radiological centers.

\section{$\underline{\text { Inclusion criteria: }}$}

High risk pregnant women such as:

- consanguineous marriage,

- prior history of fetal malformation

- positive family history of fetal anomalies
- multiple pregnancies

Exclusion criteria:

- Normal fetal biometry

- 3rd trimester pregnant women

- Pregnant women with massive oligohydramnios

Checklist of Fetal Organs at 10-16 Weeks' Screening

- Brain (midline echo, lateral ventricles, plexus chorioideus, skull shape, cerebellum)

- Neck (Normal appearance, nuchal translucency thickness)

- Face (Eyes with lensa, nasal bonea, normal profile/mandiblea, intact lips)

- spine (longitudinal and transverse view, the overlying skin)

- Heart (position, axis, four-chamber view, symmetry, pulse)

- Stomach (existence, size and shape, relation to diaphragm)

- Abdominal wall (closure, insertion of the umbilical vessels)

- Kidneys (existence, tissue texture, size, enlargement [_4 mm] of the pelvis)

- Urinary bladder (existence, size, and shape)

- Amniotic fluid (amount normal if largest pocket_9 cm)

- Long bones (Four limbs each with three segments, hands and feet with normal orientation) 
Requirements used for the ultrasound equipment in First \& second trimester scanning:

- Real-time, gray-scale, two-dimensional (2D) ultrasound

- Transabdominal (3.0:5.0) MHz, transvaginal in first trimester \& 4D ultrasound transducers

- Adjustable acoustic power output controls with output display standards

- Freeze frame and zoom capabilities

- Electronic calipers

- Capacity to print/store images

\section{Statistical analysis}

An Excel spreadsheet was established for the entry of data. We used validation checks on numerical variables and option-based data entry method for categorical variables to reduce potential errors. The analyses were carried with SPSS software (Statistical Package for the Social Sciences, version 24, SSPS Inc, Chicago, IL, USA). The normality of the data were assessed using ShapiroWilk Test. Numerical data were described as mean $\pm \mathrm{SD}$ if normally distributed; or median and interquartile range [IQR] if not normally distributed. Frequency tables with percentages were used for categorical variables. Independent Student t-test and paired t-test were used to compare parametric quantitative variables; while Mann-Whitney tests and Wilcoxon matched pairs test were used to compare nonparametric quantitative variables. Chi-square test or McNemar-Bowker tests were used to analyze categorical variables. A p-value $<0.05$ is considered statistically significant.

\section{Results}

Fifty females were included in this study. The mean age of the included women was 30.54 \pm 6.3 years old and the mean gestational age was $19.7 \pm 4.4$ weeks. The majority of the women $(36 \%)$ were G1P0, 20\% were G2P1, and $12 \%$ were G3P2. Almost $26 \%$ of the women had positive consanguinity (table 1 )

Table 1: The demographic characteristics of the included women

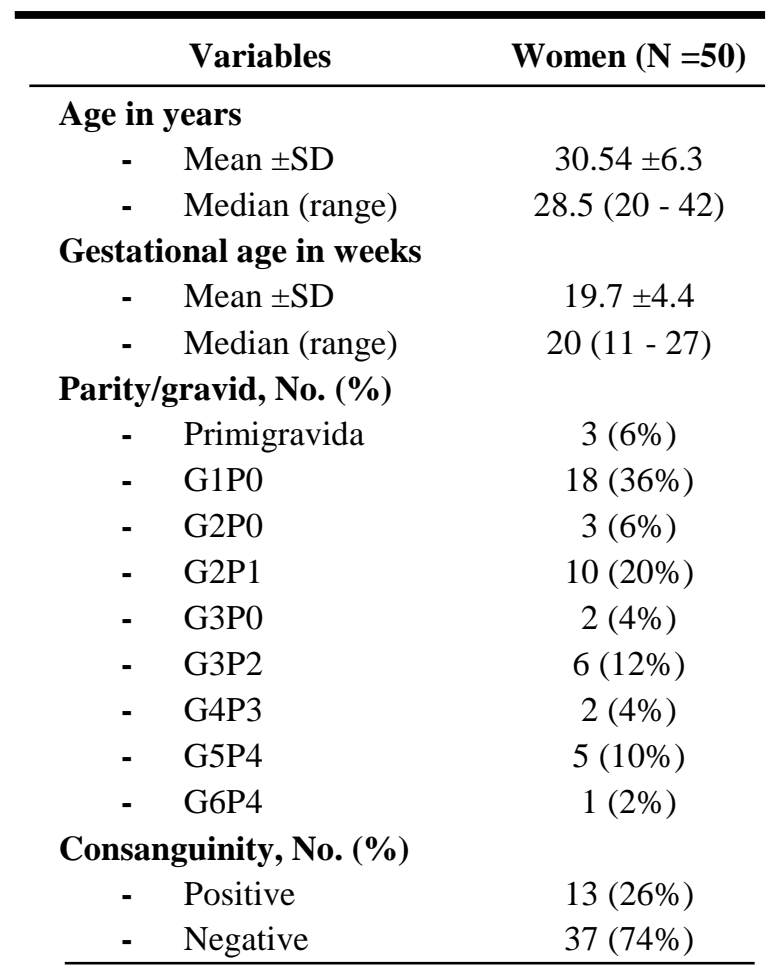

* Data are presented as mean $\pm \mathrm{SD}$, median (Range), or number $(\%)$ 
The CNS anomalies, $86 \%$ of the fetuses had normal spine, $4 \%$ had spina bifida, and $2 \%$ had arachnoid cyst, cervical minigeocele, cystic lesion, spinal mass, and spinal mixed mass normal, each.

With regard to head anomalies, 54\% had normal appearance, $6 \%$ had filated ventricle, and $2 \%$ had cardiac amorphous other one, absent cranial vault, anasarca, bananna cerebellum, brain extrusion, choidal cyst, decrease, deformed, hydrocephalus contracture, microcephaly, microcephaly, monoventricle, monoventricle microcephaly normal, neck cystic mass, and skull defect, each (Figure 1\&2).

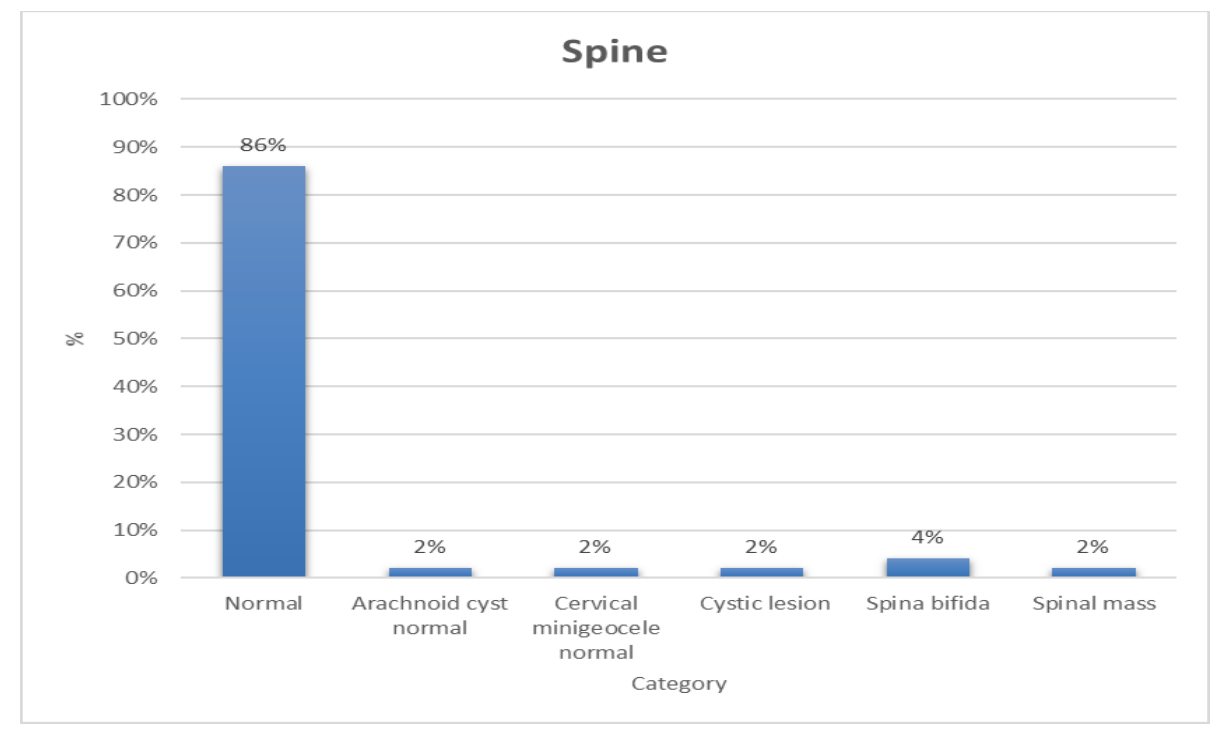

Figure 1: Distribution of spine anomalies

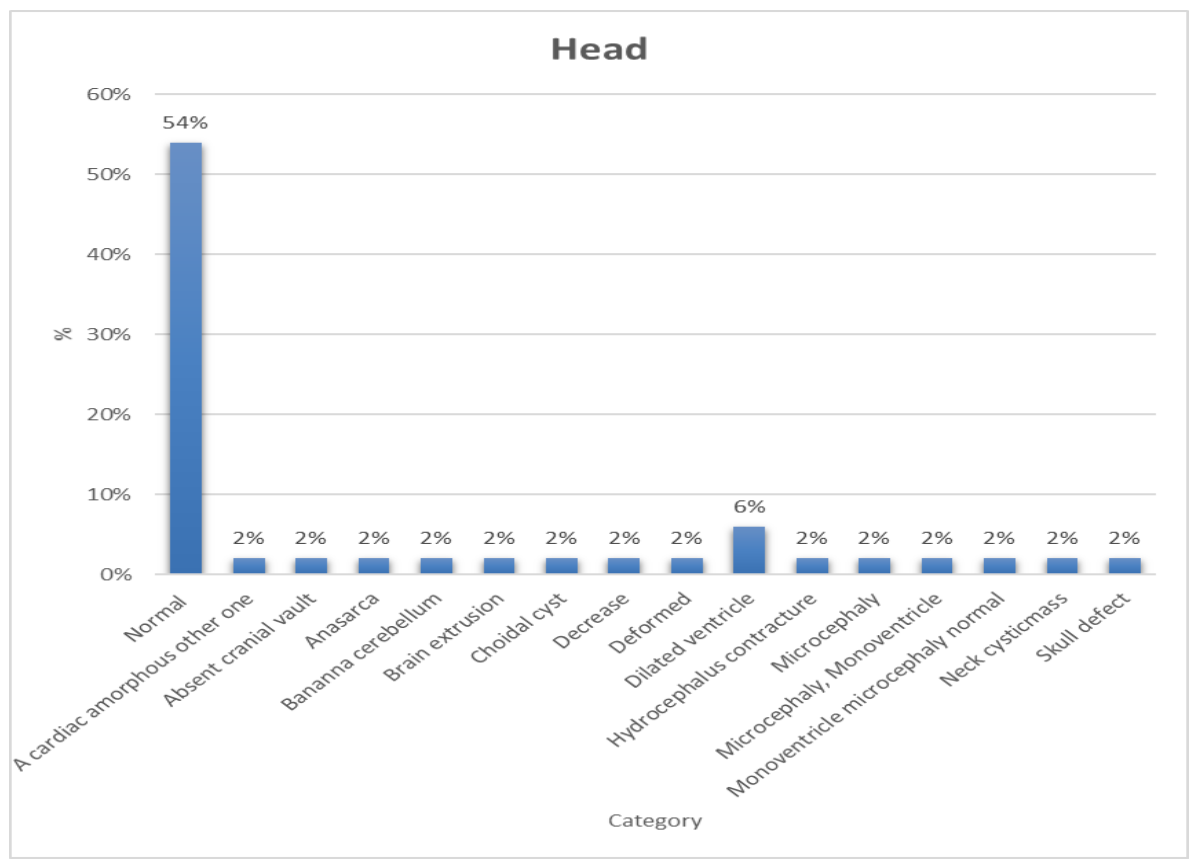

Figure 2: Distribution of head anomalies 
The facial anomalies of the included cases: $64 \%$ of the fetuses had normal appearance, $8 \%$ had cleft lip, 4\% had nasal hypoplasia, and $2 \%$ had unilateral cleft lip, macrophthalmia, microagnasia normal, and microphthalmia, each (Figure 3).
The heart anomalies: $72 \%$ of the fetuses had normal appearance and $2 \%$ had a cardiac one, atrial hypoplasia / neck mass, chest hypoplasia, deformed ribs, diaphragmatic hernia, echogenic focus in ventricles, effusion, regurge, TGA, and VSD, each (Figure 4)

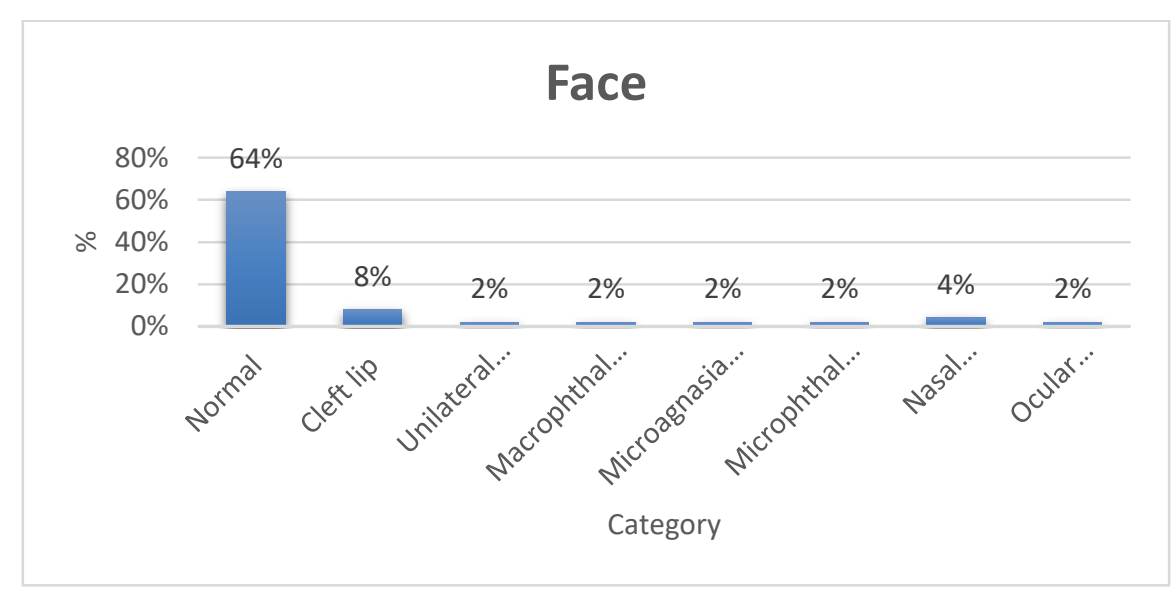

Figure 3: Distribution of face anomalies

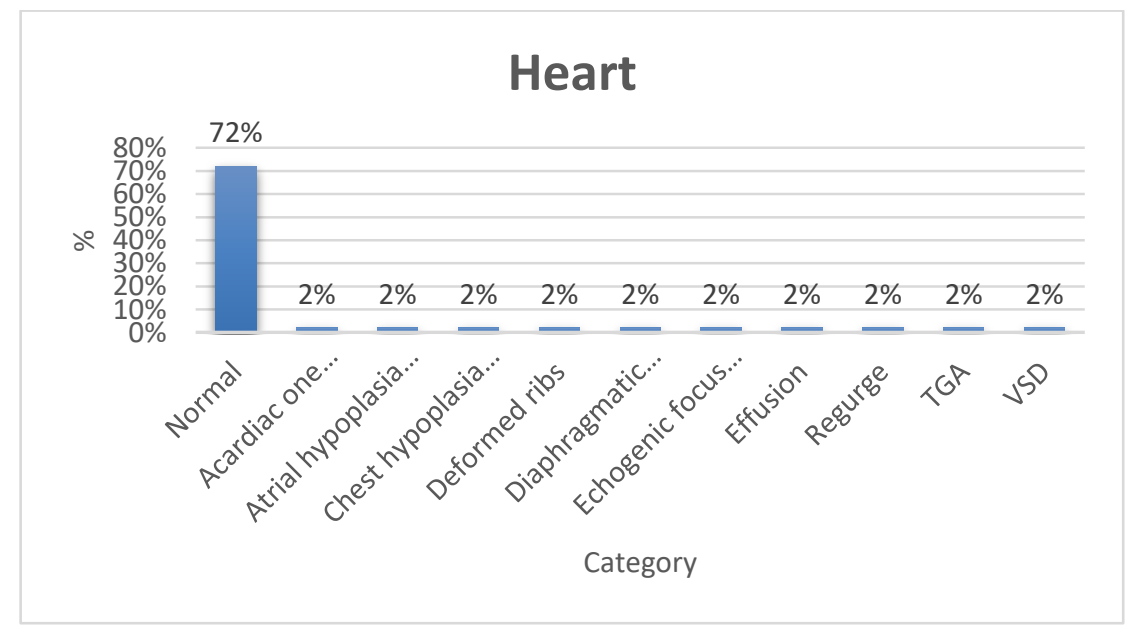

Figure 4: Distribution of heart anomalies 
The internal organ anomalies were: $88 \%$ of the fetuses had normal kidney and $2 \%$ had dilated renal pelvis average AC normal, echogenic, enlarged right kidney high AC, enlarged echogenic increase $\mathrm{AC}$, enlarged echogenic increase AC normal, and urinary bladder extrophy, each. On the other hand, $70 \%$ of the fetuses had normal abdomen, $4 \%$ had ascites, 4\% decreased AC, 4\% had hernia, and 2\% had cyst, dilated bowel loops/ normal, empty stomach, enlarged liver and spleen, and umbilical cord cyst, each (Table 2)

The upper and lower extremities anomalies were: $78 \%$ of the fetuses had normal upper limb and $10 \%$ had short upper limbs. On the other hand, $76 \%$ of the fetuses had normal hand and $4 \%$ had deformed hand. $80 \%$ of the fetus had normal lower limbs and 10\% had short lower limbs. With regard to feet, $86 \%$ had normal feet and $4 \%$ had talipes feet (Table 3)

The accuracy of 4D US in comparison to $2 \mathrm{D}$ US in the included cases was: the 2D US accurately identified $14 \%$ of the anomalies, less anomalies in $30 \%$ of the cases, and more anomalies in $30 \%$ of the cases; while 4D US had no role in $26 \%$ of the cases. On the contrary, the 4D US accurately identified $46 \%$ of the cases, less identified $24 \%$ of the cases, and more identified $30 \%$ of the cases. The difference between 4DUS and 2DUS was significantly different favoring 4D US (p $=0.001)$. Table 4

Table 2: Internal Organ anomalies of the included women

\begin{tabular}{clr}
\hline \multicolumn{1}{c}{ Variables } & \multicolumn{2}{c}{ Women (N =50) } \\
\hline Kidney, No. (\%) & 44 & $(88 \%)$ \\
- Normal & 1 & $(2 \%)$ \\
- Dilated renal pelvis average AC normal & 1 & $(2 \%)$ \\
- Echogenic & 1 & $(2 \%)$ \\
- Enlarged right kidney high AC & 1 & $(2 \%)$ \\
- Enlarged echogenic increase AC & 1 & \\
- Enlarged echogenic increase AC normal & $1(2 \%)$ & $(70 \%)$ \\
- Urinary bladder extrophy & & $(4 \%)$ \\
Abdomen, No. $(\boldsymbol{\%})$ (n =46) & 35 & $(2 \%)$ \\
- Normal & 2 & $(4 \%)$ \\
- Ascites/ AC high & 1 & $(2 \%)$ \\
- Cyst & 2 & $(2 \%)$ \\
- Decrease AC & 1 & $(2 \%)$ \\
- Dilated bowel loops $\backslash$ normal & 1 & $(4 \%)$ \\
- Empty stomach normal & 1 & \\
- Enlarged liver and spleen & 2 & \\
- Herniation & $1(2 \%)$ & \\
- Umbilical cord cyst normal & & \\
\hline
\end{tabular}


Table 3: Upper and Lower extremities anomalies of the included women

\begin{tabular}{|c|c|c|}
\hline Variables & \multicolumn{2}{|c|}{ Women $(\mathbf{N}=\mathbf{5 0})$} \\
\hline \multicolumn{3}{|l|}{ Upper Limb, No. (\%) (n =47) } \\
\hline - Normal & 39 & $(78 \%)$ \\
\hline - $\quad$ Short & 5 & $(10 \%)$ \\
\hline - $\quad$ Bowing and short & 1 & $(2 \%)$ \\
\hline - Deformed & 1 & $(2 \%)$ \\
\hline - $\quad$ Flexed & $1(2 \%)$ & \\
\hline \multicolumn{3}{|l|}{ Hand, No. $(\%)(n=43)$} \\
\hline - Normal & 38 & $(76 \%)$ \\
\hline - $\quad$ Deformed & 1 & $(2 \%)$ \\
\hline - $\quad$ Oligodactly & 2 & $(4 \%)$ \\
\hline - $\quad$ Polydactly & 1 & $(2 \%)$ \\
\hline - $\quad$ Stuby & 1 & $(2 \%)$ \\
\hline \multicolumn{3}{|l|}{ Lower Limb, No. $(\%)(n=49)$} \\
\hline - Normal & 40 & $(80 \%)$ \\
\hline - Contracture & 1 & $(2 \%)$ \\
\hline - $\quad$ Deformed & 1 & $(2 \%)$ \\
\hline - $\quad$ Flexed & 1 & $(2 \%)$ \\
\hline - $\quad$ Short & 5 & $(10 \%)$ \\
\hline - $\quad$ Talipes & $1(2 \%)$ & \\
\hline \multicolumn{3}{|l|}{ Feet, No. $(\%)(n=48)$} \\
\hline - Normal & 43 & $(86 \%)$ \\
\hline - $\quad$ Club feet & 1 & $(2 \%)$ \\
\hline - $\quad$ Contracture & 1 & $(2 \%)$ \\
\hline - $\quad$ Deformed & 1 & $(2 \%)$ \\
\hline - $\quad$ Talipes & $2(4 \%)$ & \\
\hline
\end{tabular}

Table 4: Accuracy of 4D US in comparison to 2D US of the included women

\begin{tabular}{clllll}
\hline \multirow{2}{*}{ Women $\mathbf{N}=50$} & \multicolumn{3}{c}{ 4D US } & \multirow{2}{*}{ P-value } \\
\cline { 2 - 4 } & Accurate & \multicolumn{1}{c}{ Less } & More & \\
\hline 2D US, No. (\%) & & & & \\
- & Accurate & $7(30.4 \%)$ & 0 & 0 & 0.001 \\
- & Less & $4(17.3 \%)$ & 0 & $11(73.3 \%)$ & \\
- & More & $3(13 \%)$ & $12(100 \%)$ & 0 & \\
- & No role & $9(39.1 \%)$ & 0 & $4(26.7 \%)$ & \\
\hline
\end{tabular}

\section{Discussion}

The mean age of the included women was $30.54 \pm 6.3$ years old and the mean gestational age was $19.7 \pm 4.4$ weeks. The majority of the women $(36 \%)$ were G1P0, 20\% were G2P1, and $12 \%$ were G3P2. Almost $26 \%$ of the women had positive consanguinity. Our results were in agreement with study in 2015, (5) as they reported that age of majority of the studied groups was between 25-34 years. In another study, (6), they found that most of their studied females had age between 20-34 years.

Prenatal screening provides pregnant women a non-invasive risk assessment for the most common aneuploidies. Those who are considered "high-risk" then have the option for additional diagnostic (invasive) testing. 
Prior to the $1980 \mathrm{~s}$, prenatal screening consisted of risk assessment through maternal age; however, with the advent of maternal serum biochemical analysis and ultrasound, the field of prenatal screening developed significantly (7).

An ultrasound without detailed anatomic examination is appropriate for a fetal maternal evaluation of the number of fetuses, amniotic/chorionic sacs, survey of intracranial, spinal and abdominal anatomy, evaluation of a 4-chamber heart view, assessment of the umbilical cord insertion site, assessment of amniotic fluid volume, and evaluation of maternal adenexa when visible and appropriate. The use of 3D technology can reduce scanning time while maintaining adequate visualization of the fetus in obstetrical ultrasound (8).

The present study showed that twelve percent of the included women had hypertension and $2 \%$ had preeclampsia. In addition, $18 \%$ of the women had diabetes. Other common comorbidities were convulsion (4\%), epilepsy (4\%), multiple sclerosis (4\%), and osteomalacia (4\%). As regard US Findings of the included women. Most of the women (68\%) had average liquor and 24\% had polyhydramnios. Only $10 \%$ of the women had two or more fetuses.

Jones et al (9), examined the intra- and interobserver reproducibility of 3D power Doppler (3DPD) data acquisition from women at 12 weeks gestation, which were then subsequently measured by a single observer. Women with an uncomplicated, viable singleton pregnancy were scanned between $12 \pm 0$ and $13 \pm 6$ weeks gestations with a Voluson 730 Expert. 3DPD data were acquired of the whole placenta by 2 observers: the first observer captured 2 data sets and the second a single dataset. Each data set was analyzed using VOCAL in the A plane with 9-degree rotation steps. A total of 18 low-risk women were recruited with a total of 54 data sets analyzed. The intra-class correlation coefficient (ICC) was highest for the vascular indices vascularization index (VI) and vascularization-flow index (VFI), greater than 0.75. Intra-class correlation coefficient for flow index (FI) showed moderate correlation at 0.47 to 0.65 . Bland Altman plots showed the most precise vascular index to be the FI (-15\% to $10 \%$ for inter-observer agreement). There was no bias between datasets. Prospective studies are now required to identify if this analysis tool and method is sensitive enough to recognize patients with early-onset placental dysfunction.

The current study showed that as regard CNS anomalies of the included women. $86 \%$ of the fetuses had normal spine $4 \%$ had spina bifida, and $2 \%$ had arachnoid cyst, cervical minigeocele, cystic lesion, spinal mass, and spinal mixed mass normal, each. With regard to head anomalies, 54\% had normal 
appearance, $6 \%$ had filated ventricle, and $2 \%$ had cardiac amorphous other one, absent cranial vault, anasarca, bananna cerebellum, brain extrusion, choidal cyst, decrease, deformed, hydrocephalus contracture, microcephaly, microcephaly, monoventricle, monoventricle microcephaly normal, neck cysticmass, and skull defect, each.

Our results were supported by study of Blaas et al. (10) reported an early diagnosis of spina bifida using $2 \mathrm{D}$ and $3 \mathrm{D}$ ultrasound before 10 weeks of gestation. It showed the early diagnosis of spina bifida in a case of OEIS complex (omphalocele, bladder exstrophy, imperforate anus, spina bifida) at 9 weeks of gestation. Iniencephaly is a rare neural tube defect that combines extreme retroflexion (backward bending) of the head with severe defects of the spine, associated with acrania, anencephaly and encephalocele. The prognosis for those with iniencephaly is extremely poor.

In the study in our hands, as regard facial anomalies of the included women. $64 \%$ of the fetuses had normal appearance, $8 \%$ had cleft lip, 4\% had nasal hypoplasia, and 2\% had unilateral cleft lip, macrophthalmia, microagnasia normal, and microphthalmia, each.

Cicero and her colleagues reported that the nasal bone was absent in $113(0.6 \%)$ of the 20,165 chromosomally or phenotypically normal fetuses and in $87(62.1 \%)$ of the 140 fetuses with trisomy 21. 3D sonography allows a mid-sagittal section of the fetal face to be obtained by utilizing the three orthogonal planes, and avoids the pitfall of obtaining a para-sagittal view, which could lead to false negative results. Tomographic ultrasound imaging also allows demonstration of facial midline structures in detail, by examining close parallel sections. It is important to be aware that the quality of the orthogonal planes or tomographic imaging is strongly dependent upon the original plane of acquisition (11).

The present study showed that as regard heart anomalies of the included women. $72 \%$ of the fetuses had normal appearance and 2\% had acardiac one, atrial hypoplasia / neck mass, chest hypoplasia, deformed ribs, diaphragmatic hernia, echogenic focus in ventricles, effusion, regurge, TGA, and VSD, each.

Ahmed (12), stated that CHD is the commonest congenital anomaly. It is much more common than chromosomal malformations and spinal defects. Its' estimated incidence is about 4 to 13 per 1,000 live births. Congenital heart disease is a significant cause of fetal mortality and morbidity. Antenatal diagnosis of $\mathrm{CHD}$ is extremely difficult and requires extensive training and expertise. The detection rate of CHD is very variable and it ranged from 35 to $86 \%$ in most studies. In the light of the 
above, the introduction of the new 3D/4D based STIC is highly welcomed to improve antenatal detection of CHD. Spatio-temporal image correlation is an automated device incorporated into the ultrasound probe and has the capacity to perform slow sweep to acquire a single 3D volume. This acquired volume is composed of a great number of $2 \mathrm{D}$ frames. This volume can be analyzed and reanalyzed as required to demonstrate all the required cardiac views. It also provides the examiner with the ability to review all images in a looped cine sequence. The author concluded that this technology has the ability to improve the ability to examine the fetal heart in the acquired volume and decrease examination time; it is a promising tool for the future (12).

The current study showed that as regard internal organ anomalies of the included women. $88 \%$ of the fetuses had normal kidney and $2 \%$ had dilated renal pelvis average AC normal, echogenic, enlarged right kidney high AC, enlarged echogenic increase AC, enlarged echogenic increase AC normal, and urinary bladder extrophy, each. On the other hand, $70 \%$ of the fetuses had normal abdomen, 4\% had ascites, 4\% decreased AC, $4 \%$ had hernia, and $2 \%$ had cyst, dilated bowel loops\ normal, empty stomach, enlarged liver and spleen, and umbilical cord cyst, each.
As regard upper and lower extremities anomalies of the included women. $78 \%$ of the fetuses had normal upper limb and $10 \%$ had short upper limbs. On the other hand, 76\% of the fetuses had normal hand and $4 \%$ had deformed hand. $80 \%$ of the fetus had normal lower limbs and $10 \%$ had short lower limbs. With regard to feet, $86 \%$ had normal feet and $4 \%$ had talipes feet.

Limb abnormalities can occur as isolated findings or as one component of a syndrome or sequence. However, only $5 \%$ of congenital hand anomalies occur as part of a recognized syndrome. Overlapping fingers, wrist contracture and forearm deformities are often associated with a chromosomal abnormality such as trisomy18. Most skeletal anomalies are recognizable in the second trimester. However, several reports on congenital skeletal abnormalities (such as sirenomelia and others) in the first trimester have been documented (13).

The introduction of 3D/4D sonography with high frequency transvaginal transducer has resulted in remarkable progress in ultrasonographic visualization of early embryos and fetuses and development of new fields of 3D sonoembryology. With the proper use of this new diagnostic modality and with experienced examiner, both structural and functional development in the first trimester of gestation can be assessed more objectively and reliable (14). 


\section{Conclusion}

According to our findings, 3D/4D US has shown advantage over 2DUS in demonstrating some anomalies of the face and extremities. Large studies comparing the diagnostic performance of 2DUS and 4DUS for the diagnosis of congenital anomalies have shown discordant results. However, 3D/4D US is rapidly gaining popularity as it moves out of the research environment and into the clinical setting.

That modality has advantages over 2D US, as for its recent computer technology and display techniques, and promises to play an increasingly important role in medicine. In spite of its positive psychological effect and its fascination for experts and the general public, a broad implementation of the method, for example in the form of screening tests cannot be recommended from a scientific and economic point of view.

Currently, the gold standard for evaluation of fetal anomalies remains by $2 \mathrm{D}$ US assessment, with 3D/4D US having an adjunctive role. Four dimensional US may demonstrate a more live clear image of the fetus, and only rarely adds new diagnostic information over 2DUS. However, as experience with 4D use increases and the technology itself evolves, it can be expected that its use will flourish and enhance the efficiency and accuracy of 2D US in fetal anomaly diagnosis.

\section{Sources of funding}

This research did not receive any specific grant from funding agencies in the public, commercial, or not-for-profit sectors.

\section{Author contribution}

Authors contributed equally in the study.

\section{Conflicts of interest}

No conflicts of interest

\section{References}

1. Salomon LJ, Alfirevic Z, Berghella V, Bilardo C, Hernandez-Andrade E, Johnsen SL, et al. Practice guidelines for performance of the routine mid-trimester fetal ultrasound scan. Ultrasound Obstet Gynecol. 2011;37(1):116-26.

2. Ximenes R. ISUOG Guías Prácticas: realización de la exploración ecográfica fetal del primer trimestre. Ultrasound Obs Gynecol. 2013;41:102-13.

3. Ville Y. Fetal therapy: practical ethical considerations. Prenat Diagn. 2011;31(7):621-7.

4. Barth RA. Imaging of fetal chest masses. Pediatr Radiol. 2012;42(1):62-73.

5. Khambalia AZ, Collins CE, Roberts CL, Morris JM, Powell KL, Tasevski V, et al. High maternal serum ferritin in early pregnancy and risk of spontaneous preterm birth. $\mathrm{Br} \mathrm{J}$ Nutr. 2015;114(3):455-61.

6. Bodnar LM, Platt RW, Simhan HN. Earlypregnancy vitamin D deficiency and risk of preterm birth subtypes. Obstet Gynecol. 2015;125(2):439.

7. Rao R, Platt LD. Ultrasound screening: Status of markers and efficacy of screening for structural 
abnormalities. In: Seminars in perinatology. Elsevier; 2016. p. 67-78.

8. Benacerraf BR, Shipp TD, Bromley B. Improving the efficiency of gynecologic sonography with 3-dimensional volumes: a pilot study. J ultrasound Med. 2006;25(2):165-71.

9. Jones NW, Raine-Fenning N, Mousa H, Bradley E, Bugg G. Evaluation of the intraobserver and interobserver reliability of data acquisition for three-dimensional power Doppler angiography of the whole placenta at 12 weeks gestation. Ultrasound Med Biol. 2010;36(9):1405-11.

10. Blaas $\mathrm{H}$, Eik-Nes $\mathrm{SH}$, Isaksen $\mathrm{C} \mathrm{V}$. The detection of spina bifida before 10 gestational weeks using two-and three-dimensional ultrasound. Ultrasound Obstet Gynecol Off J Int Soc Ultrasound Obstet Gynecol. 2000;16(1):25-9.
11. Cicero S, Avgidou K, Rembouskos G, Kagan KO, Nicolaides KH. Nasal bone in first-trimester screening for trisomy 21. Am J Obstet Gynecol. 2006;195(1):109-14.

12. Ahmed BI. The new $3 \mathrm{D} / 4 \mathrm{D}$ based spatiotemporal imaging correlation (STIC) in fetal echocardiography: a promising tool for the future. J Matern Neonatal Med. 2014;27(11):1163-8.

13. Monteagudo A, Mayberry P, Rebarber A, Paidas M, Timor-Tritsch IE. Sirenomelia sequence: First-trimester diagnosis with both twoand three-dimensional sonography. J ultrasound Med. 2002;21(8):915-20.

14. Pooh RK, Kurjak A. 3D/4D sonography moved prenatal diagnosis of fetal anomalies from the second to the first trimester of pregnancy. Taylor \& Francis; 2012.

To cite this article: Ahmed F. Yousef, Osama T. Galal, Taghreed H. Atta. 4D Sonography in Prenatal Diagnosis of Fetal Anomalies in First \&Second Trimester of Pregnancy. BMFJ 2020; 37(Radiology): 87-99. DOI: 10.21608/bmfj.2020.36753.1295. 